PROCEEDINGS OF THE

AMERICAN MATHEMATICAL SOCIETY

Volume 131, Number 7, Pages 2097-2102

S 0002-9939(03)07030-8

Article electronically published on February 11, 2003

\title{
BEHAVIOR OF THE BERGMAN KERNEL AND METRIC NEAR CONVEX BOUNDARY POINTS
}

\author{
NIKOLAI NIKOLOV AND PETER PFLUG \\ (Communicated by Mei-Chi Shaw)
}

\begin{abstract}
The boundary behavior of the Bergman metric near a convex boundary point $z_{0}$ of a pseudoconvex domain $D \subset \mathbb{C}^{n}$ is studied. It turns out that the Bergman metric at points $z \in D$ in the direction of a fixed vector $X_{0} \in \mathbb{C}^{n}$ tends to infinity, when $z$ is approaching $z_{0}$, if and only if the boundary of $D$ does not contain any analytic disc through $z_{0}$ in the direction of $X_{0}$.
\end{abstract}

For a domain $D \subset \mathbb{C}^{n}$ we denote by $L_{h}^{2}(D)$ the Hilbert space of all holomorphic functions $f$ that are square-integrable and by $\|f\|_{D}$ the $L_{2}$-norm of $f$. Let $K_{D}(z)$ be the restriction on the diagonal of the Bergman kernel function of $D$. It is well known (cf. [5]) that

$$
K_{D}(z)=\sup \left\{|f(z)|^{2}: f \in L_{h}^{2}(D),\|f\|_{D} \leq 1\right\} .
$$

If $K_{D}(z)>0$ for some point $z \in D$, then the Bergman metric $B_{D}(z ; X), X \in \mathbb{C}^{n}$, is well defined and can be given by the equality

$$
B_{D}(z ; X)=\frac{M_{D}(z ; X)}{\sqrt{K_{D}(z)}}
$$

where

$$
M_{D}(z ; X)=\sup \left\{\left|f^{\prime}(z) X\right|: f \in L_{h}^{2}(D),\|f\|_{D}=1, f(z)=0\right\} .
$$

We say that a boundary point $z_{0}$ of a domain $D \subset \mathbb{C}^{n}$ is convex if there is a neighborhood $U$ of this point such that $D \cap U$ is convex.

In [4], Herbort proved the following

Theorem 1. Let $z_{0}$ be a convex boundary point of a bounded pseudoconvex domain $D \subset \mathbb{C}^{n}$ whose boundary contains no nontrivial germ of an analytic curve near $z_{0}$. Then

$$
\lim _{z \rightarrow z_{0}} B_{D}(z ; X)=\infty
$$

for any $X \in \mathbb{C}^{n} \backslash\{0\}$.

Herbort's proof is mainly based on Ohsawa's $\bar{\partial}$-technique. The main purpose of this note is to generalize Theorem 1 using more elementary methods.

Received by the editors January 21, 2002.

2000 Mathematics Subject Classification. Primary 32A25.

Key words and phrases. Bergman kernel, Bergman metric.

(C)2003 American Mathematical Society 
For a convex boundary point $z_{0}$ of a domain $D \subset \mathbb{C}^{n}$ we denote by $L\left(z_{0}\right)$ the set of all $X \in \mathbb{C}^{n}$ for which there exists a number $\varepsilon_{X}>0$ such that $z_{0}+\lambda X \in \partial D$ for all complex numbers $\lambda,|\lambda| \leq \varepsilon_{X}$. Note that $L\left(z_{0}\right)$ is a complex linear space.

Then our result is the following one.

Theorem 2. Let $z_{0}$ be a convex boundary point of a bounded pseudoconvex domain $D \subset \mathbb{C}^{n}$ and let $X \in \mathbb{C}^{n}$. Then

(a) $\liminf _{z \rightarrow z_{0}} K_{D}(z) \operatorname{dist}^{2}(z, \partial D) \in(0, \infty]$;

(b) $\lim _{z \rightarrow z_{0}} B_{D}(z ; X)=\infty$ if and only if $X \notin L\left(z_{0}\right)$. Moreover, this limit is locally uniform in $X \notin L\left(z_{0}\right)$;

(c) if $L\left(z_{0}\right)=\{0\}$, then (a) and (b) are still true without the assumption that $D$ is bounded.

Proof of Theorem 2. To prove (a) and (b) we will use the following localization theorem for the Bergman kernel and metric [2].

Theorem 3. Let $D \subset \mathbb{C}^{n}$ be a bounded pseudoconvex domain and let $V \subset \subset U$ be open neighborhoods of a point $z_{0} \in \partial D$. Then there exists a constant $\tilde{C} \geq 1$ such that

$$
\begin{gathered}
\tilde{C}^{-1} K_{D \cap U}(z) \leq K_{D}(z) \leq K_{D \cap U}(z), \\
\tilde{C}^{-1} B_{D \cap U}(z ; X) \leq B_{D}(z ; X) \leq \tilde{C} B_{D \cap U}(z ; X)
\end{gathered}
$$

for any $z \in D \cap V$ and any $X \in \mathbb{C}^{n}$. (Here $K_{D \cap U}(z)$ and $B_{D \cap U}(z ; \cdot)$ denote the Bergman kernel and metric of the connected component of $D \cap U$ that contains $z$.)

So, we may assume that $D$ is convex.

To prove part (a) of Theorem 2, for any $z \in D$ we choose a point $\tilde{z} \in \partial D$ such that $\|z-\tilde{z}\|=\operatorname{dist}(z, \partial D)$. We denote by $l$ the complex line through $z$ and $\tilde{z}$. By the Oshawa-Takegoshi extension theorem for $L^{2}$-holomorphic functions 7 , it follows that there exists a constant $C_{1}>0$ only depending on the diameter of $D$ (not on $l$ ) such that

$$
K_{D}(z) \geq C_{1} K_{D \cap l}(z)
$$

Since $D \cap l$ is convex, it is contained in an open half-plane $\Pi$ of the $l$-plane with $\tilde{z} \in \partial \Pi$. Then

$$
K_{D \cap l}(z) \geq K_{\Pi}(z)=\frac{1}{4 \pi \operatorname{dist}^{2}(z, \partial \Pi)} .
$$

Now, part (a) of Theorem 2 follows from the inequalities (1), (2) and the fact that $\operatorname{dist}(z, \partial \Pi) \leq\|z-\tilde{z}\|=\operatorname{dist}(z, \partial D)$.

To prove part (b) of Theorem 2, we denote by $N\left(z_{0}\right)$ the complex affine space through $z_{0}$ that is orthogonal to $L\left(z_{0}\right)$. Set $E\left(z_{0}\right)=D \cap N\left(z_{0}\right)$. Note that $E\left(z_{0}\right)$ is a nonempty convex set. So, part (b) of Theorem 2 will be a consequence of the following:

Theorem 4. Let $z_{0}$ be a boundary point of a bounded convex domain $D \subset \mathbb{C}^{n}$. Then:

(i) $\lim _{z \rightarrow z_{0}} B_{D}(z ; X)=\infty$ locally uniformly in $X \notin L\left(z_{0}\right)$; 
(ii) for any compact set $K \subset \subset E\left(z_{0}\right)$ there exists a constant $C>0$ such that

$$
B_{D}(z ; X) \leq C\|X\|, \quad z \in K^{0}, X \in L\left(z_{0}\right),
$$

where $K^{0}:=\left\{z_{0}+t z: z \in K, 0<t \leq 1\right\}$ is the cone generated by $K$.

Proof of Theorem 4. To prove (i) we will use the well-known fact that the Carathéodory metric $C_{D}(z ; X)$ of $D$ does not exceed $B_{D}(z ; X)$. On the other hand, we have the following simple geometric inequality [1]:

$$
C_{D}(z ; X) \geq \frac{1}{2 d(z ; X)}
$$

where $d(z ; X)$ denotes the distance from $z$ to the boundary of $D$ in the $X$-direction, i.e., $d(z ; X):=\sup \{r: z+\lambda X \in D, \lambda \in \mathbb{C},|\lambda|<r\}$. So, if we assume that (i) does not hold, then we may find a number $a>0$ and sequences $D \supset\left(z_{j}\right)_{j}, z_{j} \rightarrow z_{0}, \mathbb{C}^{n} \supset$ $\left(X_{j}\right)_{j}, X_{j} \rightarrow X \notin L\left(z_{0}\right)$, such that $B_{D}\left(z_{j} ; X_{j}\right) \leq \frac{1}{2 a}$. Hence $d\left(z_{j} ; X_{j}\right) \geq a$ which implies that for $|\lambda| \leq a$ the points $z_{0}+\lambda X$ belong to $\bar{D}$ and, in view of convexity, they belong to $\partial D$. This means that $X \in L\left(z_{0}\right)$, a contradiction.

To prove part (ii) of Theorem 4, we may assume that $z_{0}=0$ and $L:=L(0)=$ $\left\{z \in \mathbb{C}^{n}: z_{1}=\ldots=z_{k}=0\right\}$ for some $k<n$. Then $N:=N(0)=\left\{z \in \mathbb{C}^{n}: z_{k+1}=\right.$ $\left.\ldots=z_{n}=0\right\}$. From now on we will write any point $z \in \mathbb{C}^{n}$ in the form $z=\left(z^{\prime}, z^{\prime \prime}\right)$, $z^{\prime} \in \mathbb{C}^{k}, z^{\prime \prime} \in \mathbb{C}^{n-k}$. Note that $L \in \partial D$ near 0 , i.e., there exists a $c>0$ such that

$$
\left\{0^{\prime}\right\} \times \Delta_{c}^{\prime \prime} \in \partial D
$$

where $\Delta_{c}^{\prime \prime} \subset \mathbb{C}^{n-k}$ is the polydisc with center at the origin and radius $c$. Since $K \subset \subset E:=E(0)$ and since $E$ is convex, there exists an $\alpha>1$ such that $K \subset \subset E_{\alpha}$, where $E_{\alpha}:=\{z: \alpha z \in E\}$. Note that $K^{0} \subset E_{\alpha}$. Using (3), the equality

$$
\left(z^{\prime}, z^{\prime \prime}\right)=\frac{1}{\alpha}\left(\alpha z^{\prime}, 0^{\prime \prime}\right)+\left(1-\frac{1}{\alpha}\right)\left(0^{\prime},\left(1-\frac{1}{\alpha}\right)^{-1} z^{\prime \prime}\right),
$$

and the convexity of $D$, it follows that

$$
F_{\alpha} \times \Delta_{\varepsilon}^{\prime \prime} \subset D
$$

where $\varepsilon:=c\left(1-\frac{1}{\alpha}\right)$ and where $F_{\alpha}$ is the projection of $E_{\alpha}$ in $\mathbb{C}^{k}$ (we can identify $E_{\alpha}$ with $\left.F_{\alpha}\right)$. For $\delta:=c(\alpha-1)$ we obtain in the same way that

$$
\tilde{D}:=D \cap\left(\mathbb{C}^{k} \times \Delta_{\delta}^{\prime \prime}\right) \subset F_{\frac{1}{\alpha}} \times \Delta_{\delta}^{\prime \prime}
$$

Now, let $(z, X) \in K^{0} \times L$. Note that $z=\left(z^{\prime}, 0^{\prime \prime}\right)$ and $X=\left(0^{\prime}, X^{\prime \prime}\right)$. Then, using (4) and the product properties of the Bergman kernel and metric, we have

$$
\begin{aligned}
M_{D}(z ; X) & \leq M_{F_{\alpha} \times \Delta_{\varepsilon}^{\prime \prime}}(z ; X) \\
& =M_{\Delta_{\varepsilon}^{\prime \prime}}\left(0^{\prime \prime} ; X^{\prime \prime}\right) \sqrt{K_{F_{\alpha}}\left(z^{\prime}\right)} \leq C_{1}\|X\| \sqrt{K_{F_{\alpha}}\left(z^{\prime}\right)}
\end{aligned}
$$

for some constant $C_{1}>0$. On the other hand, since $K^{0} \subset \subset \mathbb{C}^{k} \times \Delta_{\delta}^{\prime \prime}$, by virtue of Theorem 3 there exists a constant $\tilde{C} \geq 1$ such that

$$
K_{D}(z) \geq \tilde{C}^{-1} K_{\tilde{D}}(z) .
$$

Moreover, in view of (5), we have

$$
K_{\tilde{D}}(z) \geq K_{F_{\frac{1}{\alpha}}}\left(z^{\prime}\right) K_{\Delta_{\delta}^{\prime \prime}}\left(0^{\prime \prime}\right)
$$


and hence

$$
K_{D}(z) \geq\left(C_{2}\right)^{2} K_{F_{\frac{1}{\alpha}}}\left(z^{\prime}\right)
$$

for some constant $C_{2}>0$. Now, by (6) and (7), it follows that

$$
B_{D}(z ; X)=\frac{M_{D}(z ; X)}{\sqrt{K_{D}(z)}} \leq \frac{C_{1}}{C_{2}}\|X\| \sqrt{\frac{K_{F_{\alpha}}\left(z^{\prime}\right)}{K_{F_{\frac{1}{\alpha}}}\left(z^{\prime}\right)}} .
$$

Note that $z^{\prime} \rightarrow \alpha^{-2} z^{\prime}$ is a biholomorphic mapping from $F_{\frac{1}{\alpha}}$ onto $F_{\alpha}$ and, therefore,

$$
K_{F_{\frac{1}{\alpha}}}\left(z^{\prime}\right)=\alpha^{-4 k} K_{F_{\alpha}}\left(\alpha^{-2} z^{\prime}\right) .
$$

In view of (8) and (9), in order to finish (ii) we have to find a constant $C_{3}>0$ such that

$$
K_{F_{\alpha}}\left(z^{\prime}\right) \leq C_{3} K_{F_{\alpha}}\left(\alpha^{-2} z^{\prime}\right)
$$

for any $z^{\prime} \in H^{0}$ with $H^{0}:=\left\{t z^{\prime}: z^{\prime} \in H, 0<t \leq 1\right\}$, where $H$ is the projection of $K$ into $\mathbb{C}^{k}$ (we can identify $K$ with $H$ ).

To do this, note first that $\gamma:=\operatorname{dist}\left(H, \partial F_{\alpha}\right)>0$ since $K \subset \subset E_{\alpha}$. Fix $\tau \in(0,1]$ and $z^{\prime} \in H^{0}$, and denote by $T_{\tau, z^{\prime}}$ the translation that maps the origin in the point $\tau z^{\prime}$. It is easy to check that

$$
T_{\tau, z^{\prime}}\left(\bar{F}_{\alpha} \cap B_{\gamma}\right) \subset F_{\alpha},
$$

where $B_{\gamma}$ is the ball in $\mathbb{C}^{k}$ with center at the origin and radius $\gamma$. To prove (10), we will consider the following two cases:

Case I. $z^{\prime} \in H^{0} \backslash B_{\frac{\gamma}{2}} \subset \subset F_{\alpha}$ : Then

$$
K_{F_{\alpha}}\left(z^{\prime}\right) \leq \frac{m_{1}}{m_{2}} K_{F_{\alpha}}\left(\alpha^{-2} z^{\prime}\right),
$$

where $m_{1}:=\sup _{H^{0} \backslash B_{\frac{\gamma}{2}}} K_{F_{\alpha}}$ and $m_{2}:=\inf K_{F_{\alpha}}$.

Case II. $z^{\prime} \in H^{0} \cap B_{\frac{\gamma}{2}}$ : By Theorem 3 there exists a constant $\tilde{C}_{3} \geq 1$ such that $\tilde{C}_{3} K_{F_{\alpha}} \geq K_{F_{\alpha} \cap B_{\gamma}}$ on $F_{\alpha} \cap B_{\frac{\gamma}{2}}$. In particular,

$$
\tilde{C}_{3} K_{F_{\alpha}}\left(\alpha^{-2} z^{\prime}\right) \geq K_{F_{\alpha} \cap B_{\gamma}}\left(\alpha^{-2} z^{\prime}\right) .
$$

On the other hand, by (11) with data $T:=T_{1-\alpha^{-2}, z^{\prime}}$ it follows that

$$
K_{F_{\alpha} \cap B_{\gamma}}\left(\alpha^{-2} z^{\prime}\right)=K_{T\left(F_{\alpha} \cap B_{\gamma}\right)}\left(z^{\prime}\right) \geq K_{F_{\alpha}}\left(z^{\prime}\right) .
$$

Now, (12), (13), and (14) imply that (10) holds for $C_{3}:=\max \left\{\frac{m_{1}}{m_{2}}, \tilde{C}_{3}\right\}$. This completes the proofs of Theorem 4 and part (b) of Theorem 2 .

Remark. The approximation (5) of the domain $D \cap\left(\mathbb{C}^{k} \times \Delta_{\delta}^{\prime \prime}\right)$ by the domain $E_{\frac{1}{\alpha}} \times \Delta_{\delta}^{\prime \prime}$ can be replaced by using the Oshawa-Takegoshi theorem [7] with the data $D^{\alpha}$ and $N$. 
Finally, part (c) of Theorem 2 will be a consequence of the following two theorems.

Theorem 5 ([] $)$. Let $D \subset \mathbb{C}^{n}$ be a pseudoconvex domain and let $U$ be an open neighborhood of a local (holomorphic) peak point $z_{0} \in \partial D$. Then

$$
\lim _{z \rightarrow z_{0}} \frac{K_{D}(z)}{K_{D \cap U}(z)}=1
$$

and

$$
\lim _{z \rightarrow z_{0}} \frac{B_{D}(z ; X)}{B_{D \cap U}(z ; X)}=1
$$

locally uniformly in $X \in \mathbb{C}^{n} \backslash\{0\}$.

Theorem 6. Let $z_{0}$ be a boundary point of a bounded convex domain $D \subset \mathbb{C}^{n}$. Then the following conditions are equivalent:

(1) $z_{0}$ is a (holomorphic) peak point;

(2) $z_{0}$ is the unique analytic curve in $\bar{D}$ containing $z_{0}$;

(3) $L\left(z_{0}\right)=\{0\}$.

Note that the only nontrivial implication is $(3) \Longrightarrow(1)$. It is contained in [8]. Now, part (c) of Theorem 2 is a consequence of this implication, Theorem 5 , and part (b) of Theorem 2.

Proof of Theorem 6. The implication (2) $\Longrightarrow(3)$ is trivial.

The implication $(1) \Longrightarrow(2)$ easily follows by the maximum principle and the fact that there are a neighborhood $U$ of $z_{0}$ and a vector $X \in \mathbb{C}^{n}$ such that $(\bar{D} \cap U)+(0,1] X \subset D$ (cf. (11)).

Denote by $A^{0}(D)$ the algebra of holomorphic functions on $D$ which are continuous on $\bar{D}$. Now, following [8] we shall prove the implication $(3) \Longrightarrow(1)$; namely, (3) implies that $z_{0}$ is a peak point with respect to $A^{0}(D)$. This is equivalent to the fact (cf. [3]) that the point mass at $z_{0}$ is the unique element of the set $A\left(z_{0}\right)$ of all representing measures for $z_{0}$ with respect to $A^{0}(D)$, i.e. supp $\mu=\left\{z_{0}\right\}$ for any $\mu \in A\left(z_{0}\right)$.

Let $\mu \in A\left(z_{0}\right)$. Since $D$ is convex, we may assume that $z_{0}=0$ and $D \subset\{z \in$ $\left.\mathbb{C}^{n}: \operatorname{Re}\left(z_{1}\right)<0\right\}$. Note that if $a$ is a positive number such that $a \inf _{z \in D} \operatorname{Re}\left(z_{1}\right)>-1$ ( $D$ is bounded), then the function $f_{1}(z)=\exp \left(z_{1}+a z_{1}^{2}\right)$ belongs to $A^{0}(D)$ and $\left|f_{1}(z)\right|<1$ for $z \in \bar{D} \backslash\left\{z_{1}=0\right\}$. This easily implies (cf. [3]) that supp $\mu \subset D_{1}:=$ $\partial D \cap\left\{z_{1}=0\right\}$. Since $L(0)=0$, the origin is a boundary point of the compact convex set $D_{1}$. As above, we may assume that $D_{1} \subset\left\{z \in \mathbb{C}^{n}: \operatorname{Re}\left(z_{2}\right) \leq 0\right\}\left(z_{2}\right.$ is independent of $z_{1}$ ) and then construct a function $f_{2} \in A^{0}(D)$ such that $\left|f_{2}(z)\right|<1$ for $z \in D_{1} \backslash\left\{z_{2}=0\right\}$. This implies that supp $\mu \subset D_{1} \cap\left\{z_{2}=0\right\}$. Repeating this argument we conclude that supp $\mu=\{0\}$, which completes the proofs of Theorems 6 and 2 .

\section{ACKNOWLEDGMENTS}

This paper was written during the stay of the first author at the University of Oldenburg, supported by a grant from the DAAD. He thanks both institutions, the DAAD and the Mathematical Department of the University of Oldenburg. 


\section{REFERENCES}

[1] E. Bedford, S. Pinchuk, Convex domains with noncompact automorphism groups, Sb. Math. 82 (1995), 1-20. MR 95e:32037

[2] K. Diederich, J.E. Fornaess, G. Herbort, Boundary behavior of the Bergman metric, Proc. Symp. Pure Math. 41 (1984), 59-67. MR 85j:32039

[3] T.W. Gamelin, Uniform algebras, Chelsea, New York, 1984. Originally published by PrenticeHall, Englewood Cliffs, NJ, 1969. MR 53:14137

[4] G. Herbort, On the Bergman metric near a plurisubharmonic barrier point, Prog. Math. 188 (2000), 123-132. MR 2001g:32079

[5] M. Jarnicki, P. Pflug, Invariant distances and metrics in complex analysis, Walter De Gruyter, Berlin, New York, 1993. MR 94k:32039

[6] N. Nikolov, Localization of invariant metrics, Arch. Math. 79 (2002), 67-73.

[7] T. Ohsawa, K. Takegoshi, Extension of $L^{2}$ holomorphic functions, Math. Z. 195 (1987), 197204. MR 88g:32029

[8] N. Sibony, Une classe de domaines pseudoconvexes, Duke Math. J. 55 (1987), 299-319. MR 88g:32036

Institute of Mathematics and Informatics, Bulgarian Academy of Sciences, 1113 Sofia, Bulgaria

E-mail address: nik@math.bas.bg

Fachbereich Mathematik, Carl von Ossietzky Universität Oldenburg, Postfach 2503, D-26111 Oldenburg, Germany

E-mail address: pflug@mathematik.uni-oldenburg.de 